Mirai. Estudios Japoneses

ISSN-e: 2531-145X

http://dx.doi.org/10.5209/MIRA.60500

\title{
La poesía y la cultura del viaje: un recorrido a través del periodo clásico japonés
}

\author{
Rumi Tani Moratalla ${ }^{1}$
}

Fechas

Resumen. En este trabajo se pretende indagar sobre la cultura de viaje que había surgido en el periodo clásico de Japón, concretamente, durante el periodo Asuka y Nara mientras el proceso sociopolítico del país se consolidaba a pasos gigantescos para establecer una infraestructura de rutas marítimas y terrenales. Los motivos y las consecuencias de los viajes de ocio o "turismo" que se realizaban entre los siglos VI y VIII no difieren mucho de lo que podríamos comprender hoy en día; pero encontramos, también, aquellos viajes forzosos que empujaban al viajero indefenso a tierras desconocidas y lejos de su hogar. La emoción y la vulnerabilidad iban de la mano en esas travesías y fueron recogidas en su mayoría en la poesía. Sus testimonios más relevantes los podemos encontrar en los poemas de la antología Man'yōs$h \bar{u}$, una obra capaz de generar en la actualidad otro tipo de viaje a la inversa, el del turismo que recorre los lugares mencionados en los poemas de la antología.

Palabras clave: poemas clásicos; Man'yōshīi; cultura de viaje.

\section{[en] Poetry and Culture of Travel: A Journey through the Classical Japanese Period}

\begin{abstract}
This paper aims to investigate the culture of travel that emerged in the classical period of Japan, specifically, during the Asuka and Nara period, while the socio-political process of the country was rapidly consolidating to establish an infrastructure of maritime and terrestrial routes. The reasons and consequences of these leisure trips, or "tourism", that took place between the sixth and eighth centuries do not differ much from what we could understand today. However, there existed also forced journeys that took the helpless traveler to unknown territories, far from home. Emotion and vulnerability went hand in hand in these journeys, which were captured in poetry. The most relevant testimonies can be found in the poems of the Man'yōsh $\bar{u}$, an anthology that has been capable of generating twelve centuries later another type of journey in reverse: the tourist visitors who travel to the places mentioned in the poems in the anthology.
\end{abstract}

Keywords: classical poems; Man' 'yōshī; Culture of travel.

Sumario: Introducción. 1. Primeros viajes: los efectos sobre el terreno sociopolítico. 2. El turismo como cultura de viaje. 3. El viaje en forma de poesía. Conclusión.

Cómo citar: Tani Moratalla, R. (2018). La poesía y la cultura del viaje: un recorrido a través del periodo clásico japonés, en Mirai. Estudios Japoneses 2(2018), 129-139.

\footnotetext{
1 Universidad Autónoma de Madrid rumi.tani@uam.es
} 


\section{Introducción}

Según las palabras del antropólogo Umesao Tadao, la prioridad del viaje para los japoneses no está solo en llegar al destino, sino en disfrutar también del trayecto. El interés por el traslado del lugar habitual a otro desconocido está por detrás del interés que pueden brindarles nuevos encuentros, anécdotas inesperadas o el propio deleite al contemplar un paisaje sublime durante dicho viaje. ${ }^{2}$ Obviamente, esta opinión puede ser cuestionable en nuestros días cuando el estilo de vida ha cambiado radicalmente y se premia, sobre todo, viajar y cumplir una expectativa turística en los destinos y en un tiempo limitado. La observación de Umesao, en este sentido, podría encajar mejor en el contexto del periodo clásico japonés, concretamente en los periodos Asuka (552-710 d. C.) y Nara (710-794 d. C.), ya que fue precisamente en esta etapa cuando, por motivos sociopolíticos que veremos a continuación, el concepto de viaje como "turismo" se reforzaría gracias a las infraestructuras del gobierno de Yamato.

Los viajes durante el periodo clásico japonés presentan dos grandes particularidades: por una parte, algunos eran viajes promovidos por el interés político del Estado; $\mathrm{y}$, por otra, los que podríamos definir como viajes de turismo o de ocio, como se podría entender en la actualidad. Ambas vertientes convivieron, al parecer, desde tiempos del periodo Asuka. ${ }^{3}$ Los dos documentos oficiales e históricos que recogen estos testimonios los encontramos ya en Nihonshoki (日本書記, Crónicas de la historia de Japón) ${ }^{4}$ y Engishiki (延喜式, Compilación de normas y leyes). ${ }^{5}$ El primero, completado en el año 720 , nos presenta por orden cronológico los itinerarios y motivos del viaje de cada emperador reinante, lo que nos proporciona una fuente de información histórica relevante junto a las grandes empresas políticas realizadas por cada uno de ellos. Por otro lado, Engishiki es un texto oficial completado en el año 927 en el que se recoge la legislación y los reglamentos que conciernen al Estado y, donde hay un apartado dedicado al viaje oficial.

Junto a estas dos obras, tenemos como tercer testimonio sobre las experiencias viajeras recogidas en formato poético, más personal e íntimo, la primera antología Man'yōshu que, además de su valor lírico, nos ha dejado un interesante testimonio sobre los aspectos de la vida de los hombres y mujeres del periodo clásico, desde el siglo VI hasta el VIII. Entre su caudal de composiciones poéticas se encuentra una amplia variedad de temas y, entre ellos, el dedicado exclusivamente a los viajes.

A continuación, expondremos primero los aspectos relacionados con la idea de "viaje" y "turismo" como vía de desarrollo y, en segundo lugar, las consecuencias que el viaje tiene en el aspecto cultural, concretamente, en la poesía.

\footnotetext{
Umesao, Tadao (1974): Nihonjinno chie. Tokio: Chūō Kōronsya, pp. 173-174.

Los viajes con motivos comerciales ya se habían realizado previamente, durante los siglos IV y V.

4 Ha sido la primera obra historiográfica y cronológica, con elementos mitológicos a su vez, que recoge las empresas de los emperadores del reinado de los dioses hasta la emperatriz Jitō (645-703 d. C.).

5 El emperador Daigo fue quien encargó la elaboración de este libro en el año 905. Se fue completando hasta su conclusión en el año 927 con 20 volúmenes y, entre los 3.300 reglamentos, se recogen los asuntos del Estado, las organizaciones ministeriales y las instituciones de culto y celebración sintoísta.
} 


\section{Primeros viajes: los efectos sobre el terreno sociopolítico}

Durante el periodo clásico de Japón, el intercambio comercial fue una de las primeras prácticas de interacción socioeconómica. Al parecer, ya en el periodo Jōmon final (1000-500 a. C.), había existido cierto impulso comercial e intercambio de productos artesanales entre la región de la actual prefectura de Nagano y Aomori, separadas por una distancia de más de $500 \mathrm{~km} .{ }^{6}$ Sin embargo, el gran flujo de viajes que se convertirá en el núcleo principal para el desarrollo de la cultura de Japón surge por vía marítima a partir del siglo V d. C., a través de la península de Corea. Aunque ya había existido un trasiego en los siglos anteriores por motivos políticos y culturales, estos viajes por vía marítima se intensificaron gracias a las relaciones diplomáticas y comerciales entre la dinastía de Tang (618-907 d. C.), lo que obligó inevitablemente a crear un cuerpo de legados formado por el gobierno japonés, con el principal objetivo de importar toda la sabiduría de los distintos territorios del continente. Está documentado que entre los años 630 y 894 se habían realizado 16 misiones que embarcaron desde Japón, conocidas con el nombre de kentōshi (遣唐使). En uno de los apartados de Engishiki consta que se habían formado varias categorías según las competencias de cada miembro del grupo de legados. Entre ellas se encontraban: el primer delegado, secretarios, escribanos, médicos, técnicos de navío, estudiantes, monjes o traductores ${ }^{7}$. Durante el apogeo del imperio Tang, Japón llegó a tener unos 216 legados entre toda la región que abarcaba el reino de Shiragi (Silla) y los reinos norteños de Bohai (Balhae). Por el contrario, para los legados diplomáticos extranjeros que llegaban a Japón se habían construido pabellones de acogida, principalmente, en las ciudades costeras de Naniwa, Tsukushi y Dazaifu. ${ }^{8}$ El resultado de toda esta maquinaria de intercambio, absorción o fuerza centrípeta de la cultura china acabará desembocando en el afianzamiento sociopolítico y cultural del Estado japonés en los siglos posteriores.

Junto a la influencia foránea, los acontecimientos históricos que tuvieron lugar en Japón durante el periodo Asuka, como la reforma de Taika (Taikano kaishin) en 645 y la instauración del código de Taihō (Taihō ritsuryō) en el año 703, contribuirán a perfilar la estructura política y administrativa del gobierno japonés que se convertirá en un Estado centralizado aglutinando todo el poder administrativo en la capital de Nara. ${ }^{9}$ Inevitablemente, junto a estas rutas marítimas, surgió la necesidad de reforzar las infraestructuras viales por tierra y se completaron de esa manera las principales rutas terrestres junto con las marítimas para facilitar el tránsito por las regiones re-

\footnotetext{
Nakanishi, Susumu, (2011): Tanoshiku wakaru Man'yōshū. Tokio: Natsumesya, pp. 184-185.

Los bokkaishi 渤海使 eran delegados japoneses que viajaban entre Japón y la zona sur de la península coreana y los reinos norteños del Bohai (Balhae). Este último legado continuó activamente sus viajes incluso después de que se suspendieran los viajes de kentōshi durante la dinastía Tang en el año 894 d. C. Entre 727-926 d. C. se han registrado hasta 36 viajes de legados bokkaishi.

8 Fueron las tres ciudades portuarias más importantes de este periodo desde donde partían las naves al continente vecino.

9 Se trata de un agitado periodo histórico donde comienza a perfilarse el sistema del gobierno central tras adoptar las leyes penales y jurídicas, Ritsuryō 律令, basadas en el código administrativo y el derecho penal heredado por la dinastía Tang. Por otra parte, este periodo fue testigo también de la gran reforma política Taika no kaishin (大 化の改新) tras el asesinato de Soga no Emishi y su hijo Iruka a manos del futuro emperador Tenchi y Fujiwara no Kamatari, y que consolida el fin de la hegemonía del clan Soga y de otros clanes cercanos de la región de Asuka. Comienza así la supremacía de la figura del emperador como principal gobernante del país poniendo fin al dominio absoluto que habían disfrutado los clanes de la región central.
} 
motas del país. Como resultado, se trazaron siete rutas principales conocidas como Sichidō (七道Las siete rutas) que incluyen Tōkai (東海), Tōsan (東山) y Hokuriku ( 北陸)que conectan la región del este con la capital en la región de Nara y San-in (山 陰), Sanyō (山陽) al oeste, Nankai (南海) y Saikai (西海) con la isla de Shikoku y Kyūshū respectivamente. ${ }^{10}$

Los viajeros que por orden del gobierno central debían hacer uso de las rutas terrestres fueron principalmente funcionarios estatales que solían pertenecer a las clases altas de la sociedad; destinados a distintas regiones y provincias, ocupaban el puesto de gobernador durante un periodo determinado para después regresar a la capital. Paralelamente, también viajaban los funcionarios de rango inferior, los fueki 賦役, asignados para transportar los impuestos recaudados en las distintas regiones hasta la capital; y, por último, los guardias fronterizos, en su mayoría hombres reclutados forzosamente en sus aldeas, que debían viajar a los puestos asignados en Kyūshū, concretamente, en Tsukushi. Conocidos con el nombre de sakimori (防人), estos guardias fronterizos contribuirán involuntariamente a formar parte del volumen de poemas de viajes dentro de la antología Man'yōshū.

Por otra parte, los riesgos que podían acarrear estos viajes debido a su inseguridad, se habían reducido gracias a la infraestructura de rutas y comunicaciones establecida por el gobierno del periodo Nara, que tenía interés en garantizar la llegada de los impuestos que se recaudaban en las diferentes regiones. Para agilizar este trasiego se creó el kaso (過所), un peaje de papel o una tablilla de madera que tenía función de salvoconducto para poder atravesar todas las rutas y los puestos de guardia del país. Al mismo tiempo se construyeron estaciones de posta, umaya (駅家), cada $16 \mathrm{~km}$ a lo largo de toda la ruta. En los documentos de Engishiki consta que, a principios del siglo X, existían 403 umaya en todo el país. Estas estaciones estaban destinadas al uso exclusivo de los funcionarios de alto rango, siendo la mayoría gobernadores designados por el gobierno. Sin embargo, los viajeros de condiciones sociales más humildes carecían de estos privilegios y muchos de ellos quedaban expuestos directamente a los riesgos que amenazaban sus vidas durante la travesía, como la hambruna, las enfermedades, los asaltos, etc. Por tanto, en sus inicios, la cultura del viaje comenzó a afianzarse en Japón en el terreno administrativo y burocrático para garantizar la seguridad, al menos, del cuerpo de funcionarios de clase alta durante sus viajes.

\section{El turismo como cultura de viaje}

Si nos ceñimos a la idea de "turismo" que contiene otros propósitos alejados de los del "viaje funcional", mencionados en el apartado anterior, podemos señalar tres tipos principales de viaje que se practicaron en el periodo clásico. Los tres vienen recogidos tanto en los pasajes cronológicos en Nihonshoki como en los poemas de Man'yōshū con las siguientes denominaciones: sankei (参詣), la peregrinación o romería a los lugares religiosos, principalmente santuarios sintoístas; yūran (遊覧), que significa literalmente disfrutar en paseos itinerantes; y hoyōchi (保養地), los viajes a los baños termales por cuestiones de salud o simplemente por placer.

10 Los primeros trazos que se marcaron para las Cinco Provincias y Siete Rutas, Gokishichidō (五幾七道), tuvieron lugar durante el periodo Asuka. Posteriormente, ya en el periodo Nara, se fortalecieron estas vías cuya organización administrativa se mantuvo hasta el periodo Muromachi. 
Estos motivos se enmarcaban dentro de un patrón de viaje conocido con el nombre de gyokō (行幸) tratándose, exclusivamente, de aquellos viajes que disfrutaban los miembros de la familia imperial y la nobleza. ${ }^{11}$ El Nihonshoki recoge varios gyokō imperiales, y entre ellos tenemos uno de los gyokō más espectaculares del periodo clásico, Shigaraki gyokō (紫香楽行幸), del emperador Shōmu que reinó de 724 hasta $749 \mathrm{~d}$. C., y donde nos consta que llegaron a congregarse en ese viaje más de 2000 personas del séquito imperial, en el año $743 .{ }^{12}$

Uno de los primeros motivos del viaje sankei se debe a la coexistencia de las dos corrientes dogmáticas, el budismo y sintoísmo, que contribuyeron a crear una fusión peculiar que ha desembocado en dos tipos de viajes de peregrinación. ${ }^{13}$ Por una parte, el sintoísmo, basado en la veneración a sus yaorozuno kami (ocho millares de deidades), centraba principalmente sus cerebraciones y fiestas en torno a la siembra y la cosecha del año. Y por otra, el budismo, que además influyó en el terreno sociopolítico en los primeros gobiernos del periodo clásico japonés, también marcó un hito en la cultura de la peregrinación de los habitantes. ${ }^{14}$ Dentro de este contexto se incluyen también otros patrones de viaje que van apareciendo, como el simple deleite de contemplar y admirar el paisaje junto a los encuentros ocasionales que surgían por el camino. El motivo, aparentemente religioso, del viaje consistía en llegar a los destinos para rendir culto a una deidad, pero el mismo peso tenía ese "durante" del viaje, como puede verse plasmado en las poesías que componían los viajeros en su travesía. Salvando las distancias, lo más parecido que podríamos encontrar en la cultura española sería una "romería". ${ }^{15}$

Como contraposición a los viajes sankei se encuentra el yūran: se trataban de visitas cortas y puntuales que se realizaban en los alrededores del lugar donde acampaba la comitiva imperial. Tal como se demuestra en sus dos kanji, 遊覧, que significan apreciar y contemplar el mundo exterior con afán de diversión. Dentro de este contexto laico y menos religioso, existe una terminología que indica con claridad el objetivo e intención de los viajeros: Monomi yūzan, (物見遊山). Monomi: acudir o viajar allá donde se congrega animadamente la gente y las cosas; y yüzan: salir o viajar para cambiar de aires. ${ }^{16}$ Entre ellos se encuentra el conocido hanami yüzan ( 花見遊山), con el mismo propósito, y que está conectado con la flora del país. El popular hanami, la flor de sakura, es una acepción que se usa en el japonés actual y que incluso se considera uno de los rituales nacionales más importante del año. Los términos, como sakuragari (桜狩り), que hace referencia a recorrer los campos en busca de los sakura más espectaculares, o kanzakurakai (観桜会), consistente en

11 Debemos matizar que el gyokō o el viaje imperial, no siempre hace referencia a los viajes de larga distancia; pues la distancia podía ser relativamente corta, incluso llegando a ser una excursión de un día.

12 Takano, Masami (2009): Manyōshū no kankyō to seikatsu (万葉集の環境と生活). Tokio: Kasama syoin, p. 138.

13 Kitakawa, Mueno (2002): Kankō, tabi no bunka (観光・旅の文化). Tokio: Minerba, pp. 136-138.

14 No podremos considerar que sean muy similares a las peregrinaciones que se conocen en la cultura judeocristiana o islámica con una trayectoria lineal, que se traza desde el lugar de salida hasta el lugar del destino. En el caso de otras culturas, como la India o Japón, el trazado de la ruta suele ser circular como indica la palabra junrei (巡礼), es decir, se visitan varios templos por el camino, practicándose con una gran devoción a partir del periodo Heian.

15 La práctica de este tipo de desplazamiento por motivos religiosos, fue iniciado principalmente por los miembros de la corte imperial, cuyos viajes, llamativos y espectaculares, están recogidos cronológicamente en Nihonshoki. Entre los más conocidos se encuentran el Yoshino gyokō o Naniwa gyokō.

16 Kitakawa, Mueno (2002): Kankō, tabi..., op. cit., pp. 149-151. 
organizar encuentros con varias personas para admirar sus flores, fueron costumbres que existían desde antaño y que en Nihonshoki ya encontramos citas de este hábito en el viaje del emperador Richū. ${ }^{17}$ Estas costumbres nunca llegaron a desparecer y siguen siendo uno de los eventos más significativos hasta el día de hoy con la llegada de la primavera. De la misma manera, ese interés que suscita el cambio del entorno natural con la llegada de cada estación se ha traspasado al turismo "floral". En la actualidad existen agencias de viajes que organizan excursiones con la temática botánica o floral como puede ser el contemplar las hortensias en junio, la flor de cosmos en septiembre o las hojas de arce en noviembre.

Por otra parte, los baños termales o las aguas curativas han sido siempre otro motivo significativo en la cultura del viaje que se ha mantenido hasta hoy en la sociedad japonesa, acuñando la terminología onsen ryokou (温泉旅行) u onsen meguri (温 泉巡り), viajes a los baños termales. Se trata de un turismo con el exclusivo propósito de disfrutar de las propiedades curativas de las aguas termales. Encontramos este hábito documentado también en Nihonshoki donde, por ejemplo, consta que en septiembre del año 631 el emperador Jomei visita los baños de Arima, la actual prefectura de Hyōgo; o que la emperatriz Suimei en 658 se instaló unos meses en los parajes de las aguas curativas de Yusaki. ${ }^{18}$

\section{El viaje en forma de poesía}

La poesía ha estado estrechamente unida con las emociones que fueron naciendo en los viajes y desde esa premisa en la antología poética de Man'yōshū los poemas sobre la temática del viaje tienen su propio espacio a lo largo de sus 20 tomos.

Bajo la clasificación de kiryoka (羈旅歌), poemas de viaje o travesía, los poemas de este tema se encuentran en los tomos I, VI, VII, IX y XV, además de en el tomo $\mathrm{XX}$ donde se concentra la gran mayoría de los poemas compuestos por los sakimori, los guardias fronterizos destinados al norte de Kyūshu.

Por otra parte, encontramos entre los 4.500 poemas casi unos 1.200 topónimos que hacen referencia a distintos puntos geográficos de Japón. Partiendo de la capital de Yamato, la actual región de la prefectura de Nara, los principales topónimos se extienden hasta la isla Kyūshu al sur y a las provincias de noreste tal como nos consta en los poemas de Azuma uta (東歌), poemas de tierras del este, recogidos en el tomo XIV. La facilidad que ha permitido reunir los distintos topónimos y recogerlos en los poemas se debe, principalmente, a las rutas y caminos terrestres o marítimos que han permitido a los viajeros trasladarse de un lugar a otro.

Por otra parte, la importancia de citar los topónimos regionales en los poemas no solo obedece al deseo del poeta de introducir una estampa visual que conecte con sus emociones, sino también se busca reforzar la identidad cultural de los habitants, mencionando el nombre concreto de cada lugar. La mayoría de estos topónimos nos indica una densidad de lugares conectados con la religión sintoísta, junto a otros credos ancestrales como la veneración de las montañas sagradas, ${ }^{19}$ como pueden ser los

\footnotetext{
17 El emperador Tenchi (668-672 d. C.) fue el primero que ordenó plantar un árbol de sakura silvestre en el jardín de su palacio.

18 Kitakawa, Mueno (2002): Kankō, tabi..., op. cit., pp. 200-201.

19 Sasaki Kōmei (2006): Yamano kamito nihonjin, (山の神と日本人). Tokio: Youseisha, pp. 12-24.
} 
montes Yoshino, Hira, Takama o Mimoro; así como otros topónimos como el prado de Saga, el río Tatsuta, que se encuentran casi todos en los alrededores de la región de Nara. Estos topónimos, estrechamente relacionados con la idea de lo sagrado y la veneración, son incluidos en los poemas de género tanka o chōka, y que se combinan entre canciones de despedidas, las cuales se recitaban en los banquetes antes de la partida de un miembro del clan. El siguiente poema, por ejemplo, personifica el monte sagrado de Mimoro donde habita el dios Oomono nushi.

$\begin{array}{ll}\text { 三諸の } & \text { Del dios de Mimoro } \\ \text { 神の帯ばせる } & \text { sus aguas confluyen } \\ \text { 初瀨川 } & \text { hasta el rio Hatsuse } \\ \text { 水脈し絶えずは } & \text { Mientras persista su caudal } \\ \text { 我志れめや } & \text { jamás te olvidaré } \\ \text { Poema de despedida para el gobernador de Nagato, IX, } 1770\end{array}$

Según Itoo Hiroshi, existen tres patrones entre los motivos que se repiten en los poemas de viaje en Man'yosh $\bar{u} .{ }^{20} \mathrm{El}$ primero consiste en admirar la naturaleza desde una postura contemplativa. En el segundo, la exaltación de la nostalgia desde la distancia por la familia que se ha dejado atrás, en la ciudad o aldea. Y en el tercero, con una clara influencia de la estética poética china, la preferencia por las ruinas de una ciudad que nos recuerdan un pasado esplendoroso.

El primer patrón, aunque aparentemente refleje una postura pasiva frente a la naturaleza, esconde, en muchas ocasiones, la intención de buscar la protección de la madre naturaleza implorando el buen augurio y al mismo tiempo queriendo apaciguar a los malos espíritus que pueden influir en el trayecto. Lo podemos encontrar entre los poemas del contexto de gyokō, perteneciente a loas o alabanzas, conocido como el género sanka (讃歌) que manifiesta la grandiosidad del país gracias a logros y méritos del emperador reinante. Además, se busca la complicidad de la naturaleza para suplicar su protección, como en el siguiente tanka del cortesano Kakimoto no Hitomaro:

見れど飽かぬ
吉野の川の
常なめの
絶ゆることなく

またかけへり見む
Río de Yoshino

nunca me cansaré de contemplarte.

Igual que fluyen tus aguas sin cesar,

Solo por venir a contemplarte, mis pasos tampoco cesarán.

Kakimoto no Hitomaro, hanka de alabanza para la emperatriz Jitō, I, 317

Los poetas cortesanos más representativos de esta categoría poética son Kakimoto no Hitomaro y Yamabe no Akihito, cuya actividad creativa se sitúa, al parecer, a principio del siglo VIII. Se desconoce si se recitaban estos poemas en los banquetes palaciegos o en los grandes rituales de la corte. Aunque, otra posibilidad que se baraja es que pudieron haber sido recitados en las fiestas organizadas en las regiones y en las aldeas que recibían a la comitiva imperial.

Junto a los poemas de loa o alabanza, incluimos los poemas compuestos durante las visitas a los baños termales y a los santuarios fuertemente conectados con el sin-

20 Itoo Hiroshi (1983): Manyōno inochi (万葉の命). Tokio: Hanawa, pp. 39-41. 
toísmo; asimismo, las visitas a lugares emblemáticos por sus paisajes o por su flora como único motivo para organizar un traslado del séquito imperial y que, en tal caso, entrarían en el contexto del viaje de yuran. Así ocurre con la planta nacional más famosa, el sakura, ya que desde aquellos tiempos existía una fascinación que ha sido fuente de inspiración permanente, tal como demuestran los 41 tanka de Man 'yōshü. ${ }^{21}$

$\begin{array}{ll}\text { 桜花 } & \text { Flor de sakura, } \\ \text { 咲きかも散ると } & \text { floreces y te marchitas } \\ \text { 見るまでに } & \text { en un abrir y cerrar de ojos. } \\ \text { 誰かもここに } & \text { Como los encuentros y las despedidas, } \\ \text { 見えて散り行く } & \text { nada queda perpetuo. } \\ \text { Kakimoto no Hitomaro, XII, 3129 } & \end{array}$

Por el contrario, el ume, la flor del ciruelo, fue una flor importada de China que tuvo una buena acogida entre la élite de Japón. Muchos ordenaban plantar los ciruelos en los jardines de sus residencias y aposentos con el fin de emular la estética china, sinónimo de buen gusto. En Man'yōshū el número de poemas dedicados al ume, unos 120, supera los 41 poemas de sakura, dada la gran influencia que todavía seguía llegando desde el continente.

\section{春さえば \\ 木末隠りて \\ うぐひすそ \\ 鳴きて去ぬなる \\ 梅が下枝に \\ Settensannji no Wakamaro, V, 827}

\author{
Llegó la primavera. \\ $Y$ entre las hojas tiernas \\ un ruiseñor oculto. \\ Salta con su canto \\ entre ramas del ciruelo.
}

El segundo patrón es lo que se podría llamar la poesía de la añoranza, que vemos en el siguiente tanka anónimo. El poeta plasma en sus versos ese sentimiento de nostalgia por el hogar que dejó atrás durante el viaje:

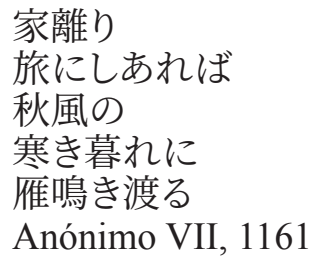

\author{
Lejos del hogar \\ mi viaje solitario. \\ En el frío atardecer \\ vuelan los gansos \\ y su graznido con el viento otoñal
}

La insistencia de querer expresar ese estado de melancolía y nostalgia que el mismo viaje causa se acentúa aún más por reunir en el mismo tanka términos como, atardecer, otoño, gansos, prototipos de un campo léxico heredado del canon poético chino que tienen la función de reforzar la tristeza. Por otra parte, los poetas muestran, dentro del contexto del viaje, una insistente nostalgia por los seres queridos que dejaron, expresando su aflicción en un entorno desconocido donde, a veces, están obligados a dormir a la intemperie, como indica explícitamente la palabra 草

${ }_{21} \quad$ El primer encuentro que se celebró para el hanami (contemplar o admirar el sakura) fue en el año 812 con el emperador Saga. 
枕 kusamakura, almohada de yerba, que establece una relación metafórica entre el contexto del entorno, el desconsuelo y la incertidumbre de la travesía.

$\begin{array}{ll}\text { 草枕 } & \text { Tú que partirás y dormirás } \\ \text { 旅行く君を } & \text { sobre la almohada de hierba. } \\ \text { 幸くあれと } & \text { Colocaré cerca de mi lecho } \\ \text { 斎聬すえつ } & \text { la sagrada vasija de vino, } \\ \text { 我が床の辺に } & \text { para que los dioses te protejan en tu travesia }\end{array}$

Sakanoue no Iratsume, XVII, 3927

Estas características abundan en aquellos tanka pertenecientes a los guardias fronterizos, sakimori, entre los que podemos encontrar el término kusamakura con más frecuencias que en otros autores. Según Sasaki, a la hora de componer un tanka de temática de viaje se pueden observar dos direcciones opuestas, determinadas por un desplazamiento: una, la que se apoya en algún topónimo o en elementos de la naturaleza que conectan con el exterior del poeta; y otra, la que hace referencia al interior de su vida personal, como a su familia o su ciudad natal. ${ }^{22}$

La construcción de rutas por tierra y las estaciones de posta que se levantaron en el periodo Nara contribuyeron a disminuir gran parte de la inseguridad vial. Pero recordemos que el uso de esas estaciones de postas estaba reservado a los funcionarios de alto rango, dado que a los guardias de frontera no se les concedía el mismo privilegio, y la mayoría debía pasar la noche a la intemperie. Muchos de ellos tenían que transportar sus propios alimentos y enseres, y esto podía hacer peligrar su vida. Los resultados podían ser nefastos, llegando incluso a perder la vida en el camino. El poeta cortesano Kakimoto no Hitomaro, testigo personal de estas desgracias, dedicó algunos de sus poemas panegíricos a los viajeros anónimos que fallecieron en sus viajes. Según Sasaki, fue el mismo Hitomaro el pionero en introducir esa imagen trágica y desconsolada de las travesías en los poemas, muy alejados de aquellos otros que exaltaban la belleza del paisaje. En otras palabras, él había creado la idea del viaje como sinónimo de riesgo y tragedia, que no necesariamente correspondía con el viaje de los altos funcionarios que sí gozaban de la garantía de poder regresar a casa, una vez finalizada la misión. De hecho, los viajeros que atravesaban el mar para viajar al continente chino llevaban consigo libros de poemas de Hitomaro como manual de inspiración para componer los suyos. Todavía más, en los banquetes palaciegos se recitaban e intercambiaban poemas de este tema, bien haciéndose pasar por viajeros expuestos al peligro, o bien exaltando la aflicción por los seres queridos a quienes no podrían volver a ver. ${ }^{23}$

En cuanto al tercer patrón, hemos mencionado que la predilección por los lugares ligados al pasado fue una herencia de la poesía china, cuya mirada nostálgica se ha asociado con las ruinas de una ciudad o de una cabaña deshabitada. En muchos de los poemas del Man'yōshū observamos una clara intención de tomar esas referencias geográficas, o de las antiguas capitales ya deshabitadas durante el viaje, sobre

Sasaki Kōmei (2006): Yamano kamito..., op. cit., pp. 102-103.

23 Sasaki corrobora también este fenómeno del género lírico dentro de la categoría de viaje que se realizaba con un motivo trágico y triste, y que contribuyó a que se desarrollara un patrón poético para recitar en los banquetes palaciegos de alta alcurnia. Esto contribuyó a mantener los modelos que se imitarán posteriormente entre los poetas, op. cit., p. 135. 
todo, para evocar la idea de melancolía por el pasado con el fin de buscar la empatía colectiva.

古の
人に我れあれや
楽浪の
古き都を
見れば悲しき
Takechi no Kurohito, I, 32

Por último, merece la pena aludir a otro término poético que tiene una estrecha relación con la cultura de viaje, el ukine (浮寝), que significa literalmente dormir flotando en el agua, tal como hacen las aves acuáticas. Principalmente aparece en los poemas compuestos durante una travesía en barco. Sasaki menciona que se ha recurrido a la palabra ukine también en los poemas de zōtōka, pertenecientes a la categoría de amor, haciendo alusión al sentimiento fluctuante o mudable que conlleva el estado del enamoramiento. ${ }^{24}$

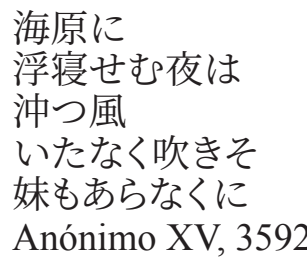

La noche en el océano, duermo en mi barca flotante. Vientos del mar no soples tan fuerte. Aquí estoy, solo sin mi amada.
No, no es posible.

Mas cuando contemplo

la antigua ciudad de Sasami, un honda pena me llena el corazón

\section{Conclusión}

La temática del viaje en los poemas clásicos japoneses se ha desarrollado en diversas vertientes debido a las circunstancias históricas del periodo clásico de Japón.

El patrón poético que se fue perfilando en los siglos VI y VIII acabó influyendo en los poetas de los siglos posteriores, ya sea en su formato de tanka o haiku.

Por una parte, hay una constante insinuación de la idea de incertidumbre, por las circunstancias que les alejaban de lo cotidiano y que ha sido el eje de los poemas de viajes compuestos por los sakimori y, posteriormente, por los poetas de la élite; por otra parte, tenemos aquellos poemas que expresaban la sincera alegría o la admiración por la belleza del entorno durante la travesía. En este último caso, causado por un viaje que estaba más próximo al concepto de "turismo" de nuestros tiempos y donde se buscaba compartir el placer y el bienestar emocional. Por tanto, estos tanka no esconden ningún elemento sombrío o inquieto que pueda transmitir el miedo del ser humano ante tierras desconocidas.

Posteriormente y, paradójicamente, los poemas de viajes del periodo clásico han inspirado a otro tipo de viaje en dirección inversa. Se trata de viajar por los parajes mencionados en dichos poemas, visitando tierras y santuarios emblemáticos como si se tratara de un guía que ayuda a los viajeros de hoy a seguir el trazo de los viajeros

24 Dentro de la antología de Man'yōshū aparecen 49 tanka con esta terminología. Sasaki Y., op. cit., p. 128. 
de otra época y lo que es más importante: compartir la misma emoción fuera de su entorno habituado.

En concordancia con esta línea y, para terminar, el presente poema es el mejor ejemplo que capta y perpetúa uno de los paisajes más emblemáticos de ayer y hoy: el monte Fuji. En este tanka se resume lo que se podría entender por cultura de viaje que surgió mientras la consolidación del gobierno de Yamato seguía su curso a grandes pasos. La expresión poética de esta temática había encajado bien en la sociedad del periodo clásico, empujada por su desarrollo politico, en cuyas rutas de viaje nacieron innumerables experiencias y emociones que quedaron fijadas para siempre.

田児の浦ゆ
うち出でてみれば
真白にそ
不尽の高嶺に
雪はふりる
Yamabeno Akihito, III, 318
Después de una travesía dejo atrás la bahía de Tago. ¡Hermosa cumbre nívea! En el monte Fuji la nieve ya había caído. 